\title{
Aspectos socioeconômicos em pacientes portadores de vícios de refração
}

\author{
Socioeconomic aspects of refractive errors
}

\author{
Alessandra Guerra Daros Castellano ${ }^{(1)}$ \\ Luciane Bugmann Moreira ${ }^{(2)}$ \\ Ticyane Teixeira Kaimoto ${ }^{(1)}$ \\ Hamilton Moreira ${ }^{(2)}$
}

\begin{tabular}{|l|}
\hline RESUMO \\
\hline Objetivo: Estudar os custos de correção dos vícios de refração em \\
grupos de pessoas de distinto poder aquisitivo. \\
Métodos: Os autores estudaram cinqüenta pacientes portadores \\
de vícios de refração. Estes foram separados em dois grupos: grupo \\
I com pacientes escolhidos de forma aleatória na primeira consulta ao \\
ambulatório de Oftalmologia do Hospital Evangélico de Curitiba \\
(HUEC), e grupo II com voluntários médicos do HUEC e acadêmicos \\
de medicina da Faculdade Evangélica de Medicina do Paraná \\
(FEMPAR). Foram analisados dados referentes a sexo, faixa etária, \\
profissão, renda, grau de instrução, uso de correção (óculos ou lentes) \\
e seu custo, consultas oftalmológicas. Os pacientes foram submetidos \\
ao exame oftalmológico de rotina. \\
Resultados: Encontramos no grupo I predominância de pacientes \\
de meia idade (48,5 anos), com renda entre 1 a 5 salários mínimos \\
(SM) e hipermétropes; e no grupo II, pacientes jovens (24,4 anos), \\
com renda acima de 20 SM e míopes foram mais freqüentes. \\
Conclusão: O gasto médio anual com óculos fica no mínimo em R\$ \\
46,50 (0,3 SM); com lentes de contato, no mínimo R\$ 196,66 (1,4 SM); \\
e com cirurgia refrativa em R\$ 800,00 (5,9 SM). O estudo sugere a \\
cirurgia refrativa como boa indicação para ambos os grupos.
\end{tabular}

Palavras-chaves: Vícios de refração; Aspectos socioeconômicos.

\section{INTRODUÇ̃̃̃O}

Sabe-se que oitenta e cinco por cento da nossa relação com o mundo exterior é feita através dos olhos ${ }^{1}$ e que tanto na fase escolar quanto no trabalho faz-se mister boa acuidade visual.

Vários procedimentos cirúrgicos foram utilizados na tentativa da cura da miopia. Desde 1890 procuraram-se métodos cirúrgicos para correção refrativa, como a epiceratoplastia, ceratotomia radial, lentes de Baikoff, ceratomileusis, anel intracorneano de Choice, fotoceratectomia refrativa(PRK), ceratectomia intraestromal a laser (LASIK). Esses fatos foram marcantes na história da cirurgia refrativa que era antes questionada pela insuficiência de metodologia científica ${ }^{2}$.

Curtin \& Whitmor ${ }^{3}$ em 1993 relataram que a prevalência de miopia atinge $20 \%$ da população mundial. Nas classes menos favorecidas o tratamento afeta o orçamento familiar e muitas vezes tais pacientes privam-se dos cuidados com a visão, o que interfere no desenvolvimento e comportamento psicossocial do indivíduo.

$\mathrm{O}$ presente estudo teve por objetivo relacionar vícios de refração e o respectivo custo de sua correção; seja com óculos, lentes de contato, e 
$11(22 \%)$ encontrados somente no grupo I, sendo que os motivos pela falta de correção foram dificuldade financeira para aquisição dos óculos em 3 pacientes, não adaptação em 1 paciente e outros como quebra dos óculos em 2, nunca ter consultado oftalmologista em 2 e sem justificativa em 3 . Os pacientes que usavam óculos corretores ou lentes de contato na primeira consulta estão demonstrados na tabela 2 .

Aferida a acuidade visual com e sem correção no melhor olho dos pacientes, tanto do Grupo I quanto do grupo II, obtivemos a maioria entre 20/20 e 20/30 conforme dados apresentados na tabela 3 .

Estão demonstrados através de gráficos (Gráfico 1) os vícios de refração encontrados nos grupos socioeconômicos analisados.

Os exames biomocroscópicos e fundoscópicos em todos os pacientes foram encontrados dentro dos padrões da normalidade exceto aquele, já citado anteriormente, com degeneração miópica em fundo de olho.

A pesquisa de preços realizada em três ópticas e serviços de lente de contato, farmácias e clínicas de cirurgia refrativa da cidade de Curitiba estão demonstrados na tabela 4 .

\section{DISCUSSÃO}

No que diz respeito aos vícios de refração causadores de baixa acuidade visual como miopia, hipermetropia e astigmatismo houve a prevalência de pacientes míopes conforme encontrado na literatura ${ }^{5}$.

No Grupo I, a maioria dos pacientes havia cursado até o $1^{\circ}$ grau. No Grupo II todos os pacientes cursavam ou haviam cursado o $3^{\circ}$ grau. Apesar deste contraste cultural, em ambos os grupos houve predomínio de pessoas inativas frente ao mercado de trabalho. Essas pessoas declararam ter renda familiar, habitar casa própria e fazer uso de correção óptica. Esses dados fazem-nos presumir que a maioria dos pacientes analisados

\begin{tabular}{|c|c|c|c|c|c|}
\hline \multicolumn{6}{|c|}{$\begin{array}{c}\text { Tabela 2. Quantidade de óculos e lentes de contato já } \\
\text { usados pelos pacientes }\end{array}$} \\
\hline \multirow{2}{*}{\multicolumn{2}{|c|}{ L. contato }} & \multicolumn{3}{|c|}{ Óculos } & \multirow[t]{2}{*}{ Total } \\
\hline & & Nenhum & 1 a 5 & 6 a 10 & \\
\hline Grupo I & 0 & 6 & 17 & 3 & 26 \\
\hline Grupo II & $8^{*}$ & 0 & 11 & 5 & 24 \\
\hline Total & 8 & 6 & 28 & 8 & 50 \\
\hline
\end{tabular}
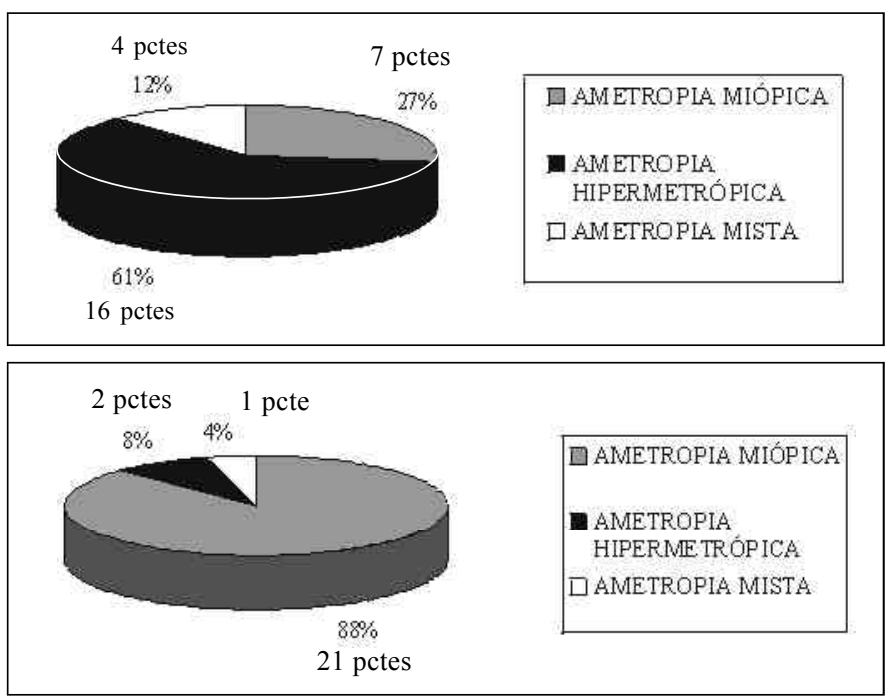

Gráfico 1. Gráficos demonstram quantidade de ametropia miópica, hipermetrópica e mista nos Grupos I e II

eram dependentes econômicamente e compartilhavam renda salarial e domicílio com seus pais, responsáveis ou outros.

Segundo o Censo ${ }^{6}$ de 1995-96 ( IBGE ), a população tem gasto mensal médio de $3,90 \%$ da renda familiar dirigida para assistência à saúde na aquisição, manutenção e cuidados com óculos e lentes de contato. Este percentual corresponde a 0,4 SM em média ao ano. Na cidade de Curitiba o par de óculos mais barato estava em torno de $\mathrm{R} \$ 46,50$ correspondente a 0,3 SM e o mais caro R\$ 1960,00 correspondente a 14,4 SM. Em um ano gasta-se em lentes de contato no mínimo $\mathrm{R} \$ 100,00$ (0,7 SM) e no máximo em torno de $\mathrm{R} \$ 500,00$ (3,6 SM), além do gasto com produtos de limpeza e conservação no mínimo de $\mathrm{R} \$ 96,66$ (0,7 SM). A cirurgia refrativa (PRK) para miopia custa R\$ 800,00 (5,9 SM) por olho, sendo a mais barata. O LASIK para astigmatismo miópico, que é a cirurgia mais cara, custa R\$1500,00 (11 SM) por olho.

Nos Estados Unidos, Chiang ${ }^{4}$, em um estudo dos aspectos socioeconômicos da cirurgia refrativa a laser, considerou o custo da cirurgia de PRK equivalente ao uso diário de lentes de contato durante 10 anos e mais viável por custo-benefício após 20 anos.

Como os óculos devem ser substituídos por novos em média a cada 2 anos, lentes de contato a cada ano e a cirurgia, teoricamente, é única, em $93,7 \%$ dos casos. A cirurgia refrativa pode ser, a longo prazo, uma solução para os pacientes do Grupo I, por serem portadores de dupla deficiência: visual e socioeconômica.

\begin{tabular}{|c|c|c|c|c|c|c|c|c|}
\hline & \multirow{2}{*}{\multicolumn{2}{|c|}{$20 / 20$ a $20 / 30$}} & \multirow{2}{*}{\multicolumn{2}{|c|}{$20 / 40$ a $20 / 80$}} & \multirow{2}{*}{\multicolumn{2}{|c|}{ Acima de $20 / 100$}} & \multirow{2}{*}{\multicolumn{2}{|c|}{ Total }} \\
\hline & & & & & & & & \\
\hline & Sem correção & Com correção & Sem correção & Com correção & Sem correção & Com correção & Sem correção & Com correção \\
\hline Grupo I & 11 & 25 & 10 & 0 & 5 & 1 & 26 & 26 \\
\hline Grupo II & 11 & 24 & 4 & 0 & 9 & 0 & 24 & 24 \\
\hline Total & 22 & 49 & 14 & 0 & 14 & 1 & 50 & 50 \\
\hline
\end{tabular}




\begin{tabular}{|c|c|c|c|}
\hline & Preços local A & Preços local B & Preços local C \\
\hline Par de óculos & $\mathrm{R} \$ 66,00 / 1960,00$ & $\mathrm{R} \$ 52,00 / 1140,00$ & $\mathrm{R} \$ 46,50 / 1716,00$ \\
\hline Lentes de contato/ano & $\mathrm{R} \$ 150,00 / 500,00$ & $\mathrm{R} \$ 120,00 / 400,00$ & $\mathrm{R} \$ 100,00 / 350,00$ \\
\hline Produtos lente/ano & $\mathrm{R} \$ 96,66 / 193,32$ & $\mathrm{R} \$ 114,00 / 192,00$ & $\mathrm{R} \$ 112,68 / 167,28$ \\
\hline Cirurgia refrativa & $\mathrm{R} \$ 1000,00 / 1500,00$ & $\mathrm{R} \$ 800,00 / 1500,00$ & \\
\hline
\end{tabular}

A cirurgia também é uma boa opção para o Grupo II, pois beneficia profissionais que atuam em condições ambientais extremas, esportistas, pacientes que não aceitam o uso de óculos, seja pela estética, seja pelo desconforto, pela anisometropia ou por não tolerarem adequadamente o uso de lentes de contato.

Qualquer tipo de correção utilizada em erros de refração é valida e tem seu espaço dentro da oftalmologia. Aumentando a diversidade de procedimentos ampliam-se as opções do paciente perante a dificuldade visual, financeira e de conforto.

\section{SUMMARY}

Purpose: To study the incidence of refractive errors in different socioeconomic populational groups and the cost of their correction.

Method: 50 patients with refractive errors were divided into 2 groups. Group I was comprised of new outpatients at the Ophthalmic Center of the Hospital Universitário Evangélico de Curitiba (HUEC). Group II consisted of doctors and medical students of HUEC who volunteered for the study. Patients'sex, age, income, educational level, visual acuity, refractive errors and expenses for their correction were recorded. All patients were submitted to ophthalmologic examination.

Results: Patients in group I averaged 48.5 years of age and a monthly income of about 5 times the Brazilian minimum wage ( $\mathrm{mw}$ ). Hyperopia was the most frequent refractive error. The corresponding findings for group II were 24.4 years of age, monthly income over 20 times mw and myopia was the most frequent.

Conclusions: Yearly expenses amounted to a minimum of 0.3 $\mathrm{mw}$ for glasses, minimum of $1.4 \mathrm{mw}$ for contact lenses and refractive surgery of $5.9 \mathrm{mw}$. We concluded that refractive surgery is a good indication for both groups in the long run.

Keywords: Refractive errors; Socioeconomic aspects.

\section{REFERÊNCIAS BIBLIOGRÁFICAS}

1 Neto SMC, Senne FMB, Matsui IA, França ST, Tanaf U, Cara JN. Levantamento do custo de óculos na cidade de Campinas SP. Revista Brasileira de Oftalmologia 1988;47:40-5.

2 Guedes A, Andrade C, Ferreira MM, Leite LAM, Ambrósio R. Ceratotomia radial. Revista Brasileira de Oftalmologia 1986;15:159-61.

3 Curtin BJ, Whitmor WG. The optics of myopia. In: TasmanW, Jaeger EA, eds. Duane's Clinical Ophthalmology. Philadelphia: JB Lippincott, 1993; v.1, capítulo 42.

4 Chiang YP, Javitt JC. The socioeconomic aspects of laser refractive surgery. Arch Ophthalmol 1994;112:1526-30.

5 Sutton G, Kalski RS, Lawless MA, Rogers C. Excimer retreatment for scarring and regression after photorefractive keratectomy for myopia. $\mathrm{Br} \mathrm{J}$ Ophthalmol 1995;79:756-9.

6 IBGE - Instituto Brasileiro de Geografia e Estatística. Pesquisa de orçamentos familiares 1995-1996. p. 205.

\section{Novidades na Internet:?!}

Agora no site CBO você tem disponível todas as informações na íntegra dos

Arquivos Brasileiros de Oitalmologia

http://w w w.cbo.com.br/abo 